\title{
Outcomes after non-cardiac surgery: mortality, complications, disability, and rehospitalization
}

\author{
Bruno Amato1, Mario Santoro², Giuseppe Giugliano3, Giuseppe Servillo4, Veronica Di Nardo5, \\ Lorenza Di Domenico5, Rita Compagna1, Raffaele Izzo² $^{2}$
}

1 Department of Clinical Medicine and Surgery, University of Naples Federico II

2 Department of Translational Medical Sciences, University of Naples Federico II

3 Department of Advanced Biomedical Sciences, University of Naples Federico II

4 Department of Neuroscience, University of Naples Federico II

5 Department of Nuclear Physics and Radiation, Guglielmo Marconi University, Rome, Italy

\begin{abstract}
In the last 25 years, the number of patients aged $\geq 75$ years undergoing non-cardiac surgery has greatly increased. In elderly patients, frailty is significantly associated with an increased risk of adverse events, functional decline, procedural complications, prolonged hospitalization, and mortality. The relationship between frailty and increased mortality and morbidity requires an appropriate tool of assessment to accurately quantify the patient's clinical and perioperative conditions. The preoperative evaluation of elderly patients candidate for non-cardiac surgery should include assessment of frailty, sarcopenia and malnutrition, as these are related to high surgical risk. For colonrectal surgery as also for gastric cancer surgery, especially early gastric cancer, the introduction of laparoscopy has yielded considerable benefits in terms of short-term postsurgical outcomes, e.g. lower rate of intraprocedural bleeding and reduced length of hospital stay. Despite the progress made in preoperative assessment, surgical procedures and postoperative management, the improvement of outcomes after noncardiac surgery in elderly patients remains a challenge and calls for future, well-designed clinical studies.
\end{abstract}

Corresponding author: Bruno Amato, Department of Clinical Medicine and Surgery, University of Naples Federico II, Via S. Pansini 5, 80131 Napoli, Italy. E-mail: bruno.amato@unina.it

Key words: Frailty; surgical outcomes; risk assessment; geriatric population.

Received for publication: 27 November 2016

Accepted for publication: 7 December 2016

CCopyright B. Amato et al., 2017

Tipografia PI-ME Editrice, Italy

Monaldi Archives for Chest Disease 2017; 87:840

doi: 10.4081/monaldi.2017.840

This article is distributed under the terms of the Creative Commons Attribution Noncommercial License (by-nc 4.0) which permits any noncommercial use, distribution, and reproduction in any medium, provided the original author(s) and source are credited.

\section{Frailty and risk of mortality}

In the last 25 years, the number of patients aged $\geq 75$ years candidate for non-cardiac surgery has greatly increased due to the exponential rise in the elderly population, accompanied by an increase in the risk of frailty. Frailty is defined as a clinical condition characterized by the presence of a state of reduced physiological reserve and a greater vulnerability to stressors. In the elderly patient, frailty is significantly associated to an increased risk of adverse events, functional decline, procedural complications, prolonged hospitalization and mortality [1]. The correlation between frailty and the increase in mortality and morbidity necessitates an appropriate assessment tool to accurately quantify the clinical and perioperative condition of the patient [2]. A number of assessment tools of frailty exist, e.g. the FRAIL scale, Cardiovascular Health Study Frailty Screening Measure (CHS), Clinical Frailty Scale, Groningen Frailty Indicator (GFI), Tilburg Frail Indicator (TFI) and Edmonton Frail Scale (EFS), and are frequently reported in the literature as valid measures of frailty. However, there exists a gap between the availability of these scales validated through clinical research and their implementation in clinical practice. The assessment of perioperative risk through the American Society of Anesthesiologists (ASA) classification [3], based on the systemic diseases affecting the patient, and the more recent guidelines of the European Society of Cardiology (ESC) concerning the assessment of preoperative cardiovascular risk do not take into account the person's level of frailty: in fact, the preoperative assessment of frailty is rarely carried out in clinical practice despite the strong evidence of its predictive value for 30-day mortality [4] and postoperative complications [5]. The assessment of frailty of the elderly patient should thus be incorporated into the perioperative setting, to evaluate patient characteristics associated with an increased risk of adverse perioperative outcomes, as a means to prevent complications and a worsening of the patient's disability.

\section{Gastrointestinal surgery}

With the ageing of the population, an increased number of elderly patients are undergoing gastrointestinal surgery. Elderly patients have a greater risk of complications during surgery for gastrointestinal neoplasm, in particular those undergoing surgery for colorectal neoplasm present a 4-fold greater risk of postsurgical complications [6]. An attentive preoperative assessment is thus necessary which includes both frailty and the syndrome of sarcopenia, characterized by a progressive 
and generalized loss of skeletal muscle mass and muscle strength, which is demonstrated to be a strong predictor of procedural complications related to interventions of gastric, esophageal, colorectal and hepato-bilio-pancreatic removal [7]. In fact, the latest guidelines of the American College of Surgeons have highlighted the importance of the assessment of frailty and of sarcopenia in the preoperative phase of a cancer surgery in patients over 75 years of age [8]. The prevalence of frailty and sarcopenia in patients affected by gastric carcinoma has been calculated to be, respectively, 30 and $38 \%$, and patients affected by esophageal and/or gastric neoplasms are at high risk of malnutrition and, thus, at very high risk of frailty.

Sheetz et al. have demonstrated a strong correlation between frailty, sarcopenia and perioperative risk of morbidity in patients undergoing esophagectomy [9]. The authors assessed the area of the psoas major through CT scans in 230 patients, showing that a normal muscle mass was associated to a greater overall survival and that the size of the psoas muscle was an independent predictor of post-surgical outcome. Obeid et al. reported that the percentage of patients affected by carcinoma of the colon undergoing surgery with subsequent grade IV or $\mathrm{V}$ complication according to the classification of Clavien was significantly higher among frail than non-frail patients, 56.3 vs $5.8 \%$ [10]. Lieffers et al. in a prospective study on 234 elderly patients who underwent resection of the colon, showed that sarcopenia was strongly associated to post-operative infections (observed in $23.7 \%$ of patients with sarcopenia vs $12.5 \%$ of patients without sarcopenia) [11]. Thus, the preoperative assessment of elderly patients undergoing gastrointestinal surgery should include the assessment of frailty and sarcopenia, both being correlated to elevated surgical risk.

\section{Improvement of outcomes in surgery for colorectal cancer}

Although treatment of colorectal neoplasm is multidisciplinary, an accurate preoperative assessment of the elderly patient and an optimal surgical strategy nevertheless represent the cornerstone for improved patient survival. Sixty-six percent of patients affected by this disease undergo at least one surgical intervention of major resection, and the pre- and perioperative care pathways are crucial to improve the clinical outcomes of surgery, such as mortality, treatment efficacy and functional recovery [12]. The preoperative assessment of frailty, sarcopenia and nutritional status in elderly patients, together with the implementation of a "personalized" surgical strategy based on their clinical conditions, have unequivocally contributed to improving the postoperative outcomes. The introduction of laparoscopic surgery has been the most significant factor in improving the short-term postoperative outcomes. In fact, laparoscopy for the treatment of neoplasms of the colon and rectum is associated to better short-term outcomes than open surgery; instead, the long-term outcomes such as survival and rate of recurrence of neoplasm of the colon, do not differ between patients who undergo laparoscopy vs open surgery. Thanks to the approach of laparoscopy, long incisions can be avoided and the surgical trauma minimized. The use of laparoscopy in the treatment of neoplasms of the colon has significantly reduced intraprocedural hemorrhage, facilitates the return to normal intestinal function and the resumption of a regular diet, and has reduced the need to administer analgesic drugs and the length of hospital stay.

Despite the improvements in the anesthesiological approach and the advances in surgical techniques, the complications after surgery for colorectal cancer have not been completely eradicated. Anastomotic dehiscence is among the most frequent and feared complications of bowel surgery. Snijders et al. published a meta-analysis in the European
Journal of Surgical Oncology in 2012, showing that $6.9 \%$ of patients who undergo bowel surgery to remove a neoplasia of the colon-rectum presented an anastomotic dehiscence which required surgical reintervention, and this complication was significantly associated to a high risk of mortality, morbidity and prolonged hospitalization [13]. Twenty percent of patients who underwent bowel surgery were malnourished, and the assessment of nutritional status through the Short Nutritional Assessment Questionnaire (SNAQ) and Malnutrition Universal Screening Tool (MUST) is of fundamental importance, particularly in the case of neoadjuvant therapy, in that the latter can induce an alteration of the metabolic and nutritional status, inducing or worsening a pre-existing state of malnutrition. Although strategies of dietary supplementation can improve the patient's muscle strength, lung function and insulin resistance, it has not been demonstrated that nutritional support yields a benefit in terms of reduced length of hospital stay or reduced incidence of anastomotic dehiscence. Thus, the assessment of the level of malnutrition through the SNAQ and MUST scores makes it possible to identify elderly patients who can benefit from dietary supplements, but it remains still to verify the predictive power of these scores for postoperative complications. Also obesity, in particular intraabdominal (visceral) obesity, is a predictor of post-operative complications, prolonged hospitalization and the higher prevalence of intraoperative conversion from laparoscopic intervention to open surgery. $\mathrm{Nu}-$ merous studies and meta-analyses have shown that programs of enhanced recovery after surgery (ERAS) have reduced the length of hospital stay and, overall, the postoperative complications [14]. There is strong evidence of the benefit in terms of postsurgical outcomes of preventive antibiotic therapy and of the preoperative preparation of the intestine, while the benefit of perioperative fluid therapy, of oxygen supplementation and of non steroid anti-inflammatory drugs (NSAIDs) remains controversial. During interventions of major surgery in which systemic hypotension and bleeding occur, there is a redistribution of blood to preserve the vital organs (heart and brain) to the detriment of the splanchnic circulation, an event which increases the risk of anastomotic dehiscence, in that an adequate perfusion of the tissues and oxygenation are fundamental for the healing of the anastomosis. Regarding perioperative fluid therapy, a restrictive therapy is associated to reduced cardiopulmonary complications compared to the administration of high quantities of fluid, bearing in mind that both therapeutic approaches can induce hypoperfusion of the anastomoses due, respectively, to hypovolemia or local edema; it has thus been proposed to assess the cardiac output to establish the quantity of fluids that need to be administered in order to prevent anastomotic hypoperfusion, an event associated to increased mortality and increased length of stay in hospital.

\section{Improvement of outcomes in surgery of stomach cancer}

Gastric cancer is generally a neoplasm with poor prognosis, and the surgical treatment of this disease is considered a high-risk procedure. The introduction of an accurate preoperative assessment of the surgical risk, which includes the Charlson Comorbidity Index (CCI) and the assessment of nutritional status and frailty, has led to an improvement in postoperative outcomes. The CCI classifies the comorbidities that have an impact on the mortality risk, and it has been validated in the surgical setting as a predictor of mortality in patients undergoing gastric surgery. It has been shown that patients in their $80 \mathrm{~s}$ and $90 \mathrm{~s}$ undergoing radical gastrectomy with $\mathrm{CCI} \geq 5$ have a greater mortality rate than elderly patients with $\mathrm{CCI}<5$. The assessment of nutritional status is also an important preoperative procedure in that stomach cancer is 
a disease at high risk of malnutrition: in fact $30-38 \%$ of affected patients present $>10 \%$ weight loss in the previous 6 months due to factors related to this type of neoplasm, such as early satiety, organ obstruction and metabolic alterations. As for colorectal neoplasms, malnutrition increases the risk of morbidity and mortality post-surgery for gastric cancer. A new strategy to improve post-surgical outcomes is the so-called 'prehabilitation', i.e. the preoperative correction of a reduced functional, nutritional, physical and neurophysiological status of the patient. This type of intervention could prove particularly useful in elderly patients, who are often impaired in these areas. As with colorectal surgery, also for the surgical treatment of gastric neoplasms, in particular for early gastric cancer, the introduction of laparoscopy has led to significant benefits in terms of postsurgical outcomes, such as a smaller intraprocedural blood loss, less postoperative pain, reduced length of hospital stay and lower levels of serum markers of postoperative stress (PCR, number of leukocytes and interleukin-6 dosed at 7 days post-surgery). The role of laparoscopy in performing a total gastrectomy remains controversial in that it is a technically very demanding procedure and it does not appear to yield significant improvements in terms of 5-year outcomes with respect to open surgery.

\section{Discussion}

The ASA classification for the assessment of perioperative risk and the ESC guidelines for the preoperative assessment of cardiovascular risk do not take into account the patient's frailty. Assessment scales of frailty (FRAIL scale, Clinical Frailty Scale, Groningen Frailty Indicator) are little used in clinical practice, despite the evidence concerning their validity and utility. In elderly patients, frailty is a strong predictor of procedural complications, adverse events, functional decline, prolonged hospitalization and mortality, and thus it is desirable that the assessment of frailty be part of the preoperative assessment of elderly patients who undergo non-cardiac surgery. Patients affected by gastroesophageal neoplasm have a high risk of malnutrition and frailty. The guidelines of the American College of Surgeons have highlighted the utility of the assessment of frailty and sarcopenia in the preoperative phase in patients over 75 years of age, sarcopenia being associated to complications during interventions of gastric, esophageal, colorectal and hepato-bilio-pancreatic resection. Laparoscopic surgery for colorectal carcinoma has clearly improved the short-term outcomes with respect to open surgery, reducing in particular the intraprocedural bleeding and the length of hospital stay, resulting thus as the surgical approach of choice when the clinical picture consents it.

\section{Conclusions}

Despite the progress made in preoperative assessment, surgical procedures and postoperative management, the improvement of outcomes post intervention in non-cardiac surgery of the elderly patient represents, still today, a field that requires future, well-designed clinical studies.

\section{References}

1. Schiattarella GG, Perrino C, Magliulo F, et al. Physical activity in the prevention of peripheral artery disease in the elderly. Front Physiol 2014;5:12.

2. Brevetti G, Giugliano G, Oliva G, et al M. The impact of comorbidity burden on the cardiovascular risk in the Peripheral Arteriopathy and Cardiovascular Events study. QJM 2008;101:575-82.

3. ASA Classification of Physical Status 2014. Available from: http:/www.asahq. org/resources/clinical-information/asa-physicalstatus-classification-system.

4. Revenig LM, Master VA, Ogan K, et al. Report of a simplified frailty score predictive of short-term postoperative morbidity and mortality. J Am Coll Surg 2015;220:904-11.

5. Beggs T, Serehri A, Szwajcer A, et al. Frailty and perioperative outcomes: a narrative review. Can J Anaesth 2015;62:143-57.

6. Tan KY, Kawamura YJ, Tokomitsu A, Tang T. Assessment for frailty is useful for predicting morbidity in elderly patients undergoing colorectal cancer resection whose comorbidities are already optimized. Am J Surg 2012;204:139-43.

7. Wagner D, DeMarco MM, Amini N, et al. Role of frailty and sarcopenia in predicting outcomes among patients undergoing gastrointestinal surgery. World J Gastrointest Surg 2016;8:27-40.

8. Chow WB, Rosenthal RA, Merkow RP, et al. Optimal preoperative assessment of the geriatric surgical patient: a best practices guideline from the American College of Surgeons National Surgical Quality Improvement Program and the American Geriatrics Society. J Am Coll Surg 2012; 215:453-66.

9. Sheetz KH, Zhao L, Holcombe SA, et al. Decreased core muscle size is associated with worse patient survival following esophagectomy for cancer. Dis Esophagus 2013;26:716-22.

10. Obeid NM, Azuh 0, Reddy S, et al. Predictors of critical care-related complications in colectomy patients using the National Surgical Quality Improvement Program: exploring frailty and aggressive laparoscopic approaches. J Trauma Acute Care Surg 2012;72:878-83.

11. Lieffers JR, Bathe OF, Fassbender K, et al. Sarcopenia is associated with postoperative infection and delayed recovery from colorectal cancer resection surgery. Br J Cancer 2012;107:931-6.

12. van Vugt JLA, Reisinger KW, Derikx JPM, et al. Improving the outcomes in oncological colorectal surgery. World J Gastroenterol 2014; 20:12445-57.

13. Snijders HS, Wouters MW, van Leersum NJ, et al. Meta-analysis of the risk for anastomotic leakage, the postoperative mortality caused by leakage in relation to the overall postoperative mortality. Eur J Surg Oncol 2012;38:1013-9.

14. Zhuang CL, Ye XZ, Zhang XD, et al. Enhanced recovery after surgery programs versus traditional care for colorectal surgery: a meta-analysis of randomized controlled trials. Dis Colon Rectum 2013;56:667-78.

15. Tegels JJW, De Maat MFG, Hulsewé KWE, et al. Improving the outcomes in gastric cancer surgery. World J Gastroenterol 2014;20: 13692-704. 